\title{
Modal evaluation of fluid volume in spacecraft propellant tanks
}

\author{
Stephanie Finnvik $^{1}$, Steven Metallo ${ }^{1}$, John Robinson ${ }^{1}$, Kevin M. Crosby ${ }^{1, \dagger}$, Rudy Werlink ${ }^{2}$ \\ ${ }^{1}$ Carthage College, Kenosha, WI, USA \\ ${ }^{2}$ NASA Kennedy Space Center, Florida, USA (rudy.werlink-1@ @asa.gov) \\ ${ }^{\dagger}$ Corresponding author: kcrosby@ carthage.edu
}

\begin{abstract}
A novel, real-time method of determining the volume of propellant present in a spacecraft tank in reduced gravity is presented. In the experiment reported here, modal analysis techniques are applied to detect shifts in resonant mode frequencies of a fluid-loaded tank as fluid is drained from the tank. These experiments are conducted in the weightless environment of parabolic flights through participation in the NASA Systems Engineering Exploration Discovery (SEED) program. Ground testing of the experimental methods is compared with the flight data, and the resolution of the analysis is estimated to be better than $10 \%$ of actual fill-fraction. Comparison with existing direct and indirect techniques for assessing propellant volume in reduced gravity suggests that the modal analysis method may prove to be more cost effective and afford better resolution than existing methods.
\end{abstract}




\section{Introduction}

Direct measurement of fluid volume in spacecraft propellant tanks is a significant challenge in microgravity environments [Fortescue et al., 2003]. In reduced gravity, traditional buoyancy or level-based techniques for fuel gauging are not useful, necessitating the use of indirect methods such as those based on equations of state (for pressurized propellant systems), impulse-response measurements of spacecraft motion, and even careful bookkeeping of thruster burn times to estimate total fuel consumption. Many of these methods require additional hardware for tank pressurization or additional propellant burns to monitor spacecraft dynamics in order to infer mass. With launch costs reaching $\$ 10,000$ per pound, uncertainty in fuel consumption is an extremely important consideration in the economics of spaceflight [Peters, 2004].

In the study reported here, we employ experimental modal analysis (EMA) to infer fluid volume in a model tank over a range of fill levels. Structural resonant frequencies shift with fluid loading as the effective mass and/or the stiffness of the structure is affected by the presence of fluid. These shifts in resonant frequency can be measured and monitored using standard techniques of EMA.

\section{Background}

Modal analysis techniques involve the application of acoustic forces to test structures. The input forces can be in the form of point sources such as impact hammers or can be presented as continuous, broad spectrum excitations such as noise or chirp functions. Natural resonances of the test structure will be excited by particular frequencies present in the the applied force, and sensors affixed to the structure record the amplitude of the acoustic response across the range of resonating frequencies.

Typically, EMA involves the computation of frequency response functions (FRF) to determine the resonant frequencies present at each sensor location. The FRF is computed as the ratio of the Fourier Transform of an output sensor to the Fourier Transform of the input signal. In this way, the FRF shows peaks at the frequencies where the sensor records a strong resonance that is not present in the spectrum of the input signal. In practice the Fourier Transforms are calculated using Fast Fourier Transform (FFT) algorithms. Modal techniques can therefore be used as real-time diagnostics of structural properties. In practice, a "monitor sensor" is positioned near the actuator to record the driver signal produced by the actuator, and is used instead of the input signal to construct the FRFs. Additional sensors are placed at different locations on the test item as discussed in Research Objectives below.

Mathematically, we denote the time series recorded by the monitor sensor as $x_{0}(t)$, and the time series recorded by the $\mathrm{i}$-th $(i=1,2, \ldots n)$ sensor as $x_{i}(t)$. The Fourier transforms of these voltage signals in frequency-space are denoted by $X_{i}(\omega)$. In terms of the transforms, the FRF of the $\mathrm{i}$-th sensor data is computed according to

$$
H_{i}(\omega)=\frac{X_{i}(\omega)}{X_{0}(\omega)} .
$$

$H_{i}(\omega)$ is a complex valued function, but here we consider only its real part which provides the information on resonance amplitudes. Data from multiple sensors $(n \geq 2)$ can be used to reconstruct geometric properties of the tank. In our case, we are less interested in the known geometric properties of the tank than in tracking frequency shifts of the primary modes.

EMA has long been used to infer the existence, location, and extent of physical defects 
such as fractures in a load-bearing column or beam [Pandey et al., 1991]. These techniques are also widely used in the component design process in the automotive and aerospace industries. More recently, modal techniques have been successfully used in the characterization of fluid-filled structures such as water tanks subject to the shear forces associated with earthquakes [Malhotra et al., 2000].

\section{Research Objectives}

The central objective of the present study is to demonstrate the feasibility of real-time, noninvasive modal analysis to determine propellant volume contained in a model spacecraft tank subject to sloshing in a zero-g environment. Fluid in a container may have two opposing influences on the structural resonances of the container. In some cases, it is possible that fluid loading of structures serves to increase the effective stiffness of the structure, an effect that would result in an increase in the structure's resonant frequencies. It is also possible that fluid loading will increase the effective mass of the loaded structure, resulting in a decrease in the structure's resonant frequencies. Whether fluid in a tank serves to increase or decrease structural resonant frequencies is determined by the coupling of fluid properties and the elastic moduli of the tank material. Extensive groundtesting performed on the tank in this study and on other tanks at NASA KSC suggests that the dominant effect of fluid loading with water and other propellant simulants is to increase the effective structural mass, thereby decreasing resonant mode frequencies.

The zero-g data was obtained through participation in NASA's Systems Engineering Exploration Discovery (SEED) student microgravity parabolic flight program. In order to assess the feasibility of modal techniques in this applica- tion, we have established preliminary estimates of the resolution of the technique in $1-\mathrm{g}$, and carried out a statistical analysis of zero-g modal data at several fill fractions. Our working hypothesis for this project is that the the effective mass of the tank is correlated most closely with the total contact area between fluid and tank wall surface (provided a minimum thickness of the fluid layer). The sloshing and swirling of the fluid in reduced gravity will result in variation of total contact area between fluid and tank walls, and therefore in a fluctuation of the resonant frequencies around the corresponding flat-surface 1-g values obtained in lab testing. We therefore expect to have to average over time windows that are much longer than the typical slosh periods. For our tank, we estimate slosh periods on the order of one second, so that our FRF time averaging will need to take place over multiple 1.0-sec. windows.

Finally, we identify experimental modes present in our data using the structural shell theory of Soedel [Soedel, 1981]. Identification of experimental modes in terms of shell theory is necessary in order to study the influence of structural artifacts such as mounting brackets and weld seams on the tank's modal response. Mode identification is also necessary to understand the optimal placement of sensors because each mode represents the unique superposition of longitudinal (wave propagation up and down the length of tank) and radial vibrations. For this reason, transverse modes are typically identified by two mode numbers referencing each of the two characteristics.

\section{Experimental Design}

Our experimental rig consists of a cylindrical steel tank of diameter $15.3 \mathrm{~cm}$, and total length including two (approximately) hemispherical end caps of $48.3 \mathrm{~cm}$. The tank has a 
capacity of two gallons, and has six FPT ports to which are attached pressure gauges, drain and fill valves, and a pressure-release valve. The tank has two welded feet for attachment to a support structure. Fig. 1 shows the tank in schematic. In the experiments reported here, the tank is oriented vertically.

Affixed to the tank are three PZT-based MFC sensors and an actuator of identical design [Smart Materials, 2011]. The actuator is driven with a white noise acoustic signal generated in LabView software from a random function sampled at $24 \mathrm{kB} / \mathrm{sec}$, amplified and biased over the voltage range -100 to $350 \mathrm{~V}$ [National Instruments, 2011]. The actuator is capable of faithfully reproducing the driver signal over the frequency range $0-10 \mathrm{kHz}$ with average power generation of $20 \mathrm{~W}$. A single sensor placed adjacent to the actuator responds directly to the actuator signal and is identified as the monitor sensor. Two additional sensors placed at empirically determined anti-nodal positions on the tank convert structural response vibrations to proportional voltage signals for processing in a data acquisition rig. The two response sensors and the monitor sensor comprise the data stream for the experiment, and their signals are sampled by a data acquisition box at $16 \mathrm{kB} / \mathrm{sec}$ to ensure that the Nyquist frequency lies well above the upper end of the frequency range of interest, $0-5 \mathrm{kHz}$. The schematic relationship of electronic components in the experimental rig is illustrated in Fig. 2.

Our team designed a custom software interface for the automated acquisition of data through the use of LabView. The interface allows for the control of data file generation and storage, pump and valve control, and DAQ settings. Design of the interface was complicated by the need to ensure that operators could manage the experiment in the challenging environment of weightlessness. Simple interface operations such as pushing buttons and typing commands that are taken for granted in lab are exceedingly difficult in zero-g environments.

In flight configuration, the experimental tank is initially $70 \%$ full with water. A softwaretriggered solenoid valve opens to drain a fixed amount of fluid from the tank into a reservoir through a small pump. We chose to keep the same fill-level for a minimum of five consecutive parabolas before draining down to next targeted fill-level. In this way, we obtained enough data at each fill-level to ensure reasonable statistical validity of the resonant frequency shifts. Fill levels are monitored by means of a flow-rate meter and post-flight calculations based on the volume of fluid contained in the reservoir tank.

\section{Results}

Fig. 3 shows a typical FRF spectrum recorded for the empty tank in 1-g lab testing. Several prominent resonances are present, with the fundamental mode at $740 \mathrm{~Hz}$. The data were acquired with a 1 second window at a sampling rate of $16 \mathrm{kB} / \mathrm{sec}$. The frequency resolution is 1.0 Hz. As sensor positions are varied along the length of the tank, the fundamental resonance at $740 \mathrm{~Hz}$ varies significantly in amplitude, disappearing entirely at nodal locations.

In Fig. 4, FRFs are recorded across several representative fill fractions with the fluid in equilibrium under 1-g lab testing. The frequency of each of the primary resonances decreases with increasing fill fraction from 0 to $70 \%$. These data confirm that the dominant role played by the fluid is one of mass-loading. The structure's effective mass increases with fluid load, resulting in a continuous decrease in the frequencies of the primary resonances of the tank. It is also important to note that the amplitude of the fundamental resonance (inset of Fig. 4) does not decrease with increasing fill fraction. 

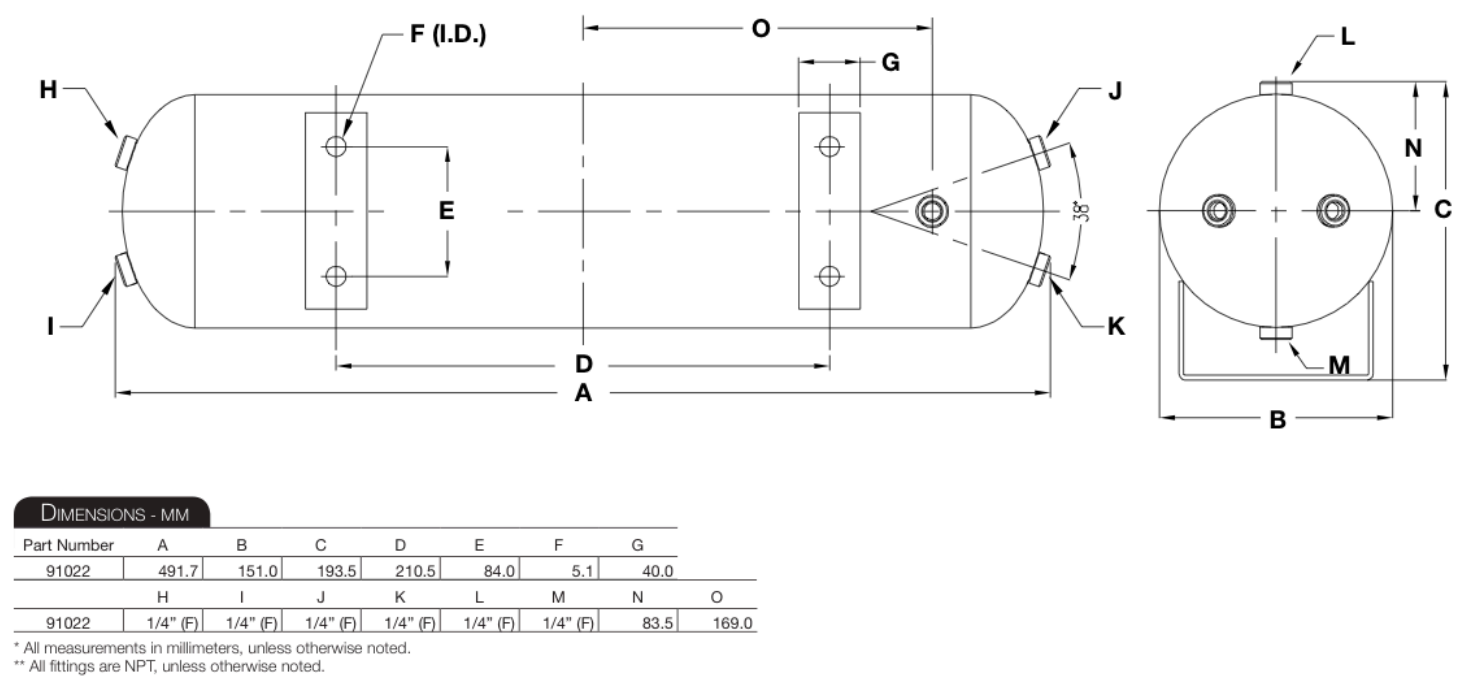

Figure 1: Schematic of experimental tank [Viar, 2011].

In the reduced gravity of the parabolic flight, fluid instabilities cause the fluid to swirl and slosh throughout the tank for periods that exceed the 30 second parabola time. The fluid is therefore in constant motion, and the sloshing results in continual variation of the contact area between fluid and tank wall. As a result, the frequencies of resonant modes will shift with periods on the order of the average slosh period of 1-2 seconds. To obtain reliable average peak positions at each fill-fraction, we choose to average multiple 1.0 second windows of FRF data to obtain average peak positions. A set of sample averaged FRFs is shown in Fig. 5. The effect of the sloshing is evident in the increased noise and reduced amplitude of the mode peaks in Fig. 5 .

To address the central question of the feasibility of the modal analysis technique for establishing the fluid volume in the tank, we extract the positions of the fundamental mode at each known fill-fraction. These data for both ground and flight data are presented in Fig. 6. The flight data in Fig. 6 have been averaged over between 5-20 1.0-second windows, resulting in a frequency resolution that is better than $10 \%$ in fill fraction. The resolution of the method may in fact exceed the $10 \%$ we estimate, but flight data was only obtained for four different fill fractions; a better assessment of mode shift resolution will require additional flight data.

\section{Comparison with Shell Theory}

To identify the modes present in the FRF data, we turn to Soedel's theory of shell vibrations [Soedel, 1981]. In the context of this theory, we consider a simply supported thin, cylindrical shell, open at both ends, and simply supported at its ends. The free vibrations of a simply supported shell can be characterized by three mode numbers, $(l, m, n)$. Here $l$ references the oscillation type (transverse, in-plane). We consider the $l=0$ modes where transverse component of oscillations dominates as these modes are an order of magnitude lower than the higher-order (shear and in-plane) $l$ modes. These higher order modes are not observed in the experimental data. The mode number $m$ characterizes the longitudinal vibrations that propagate along the length of the cylinder, while $n$ characterizes the circumferential vibrations. Therefore, we are in- 


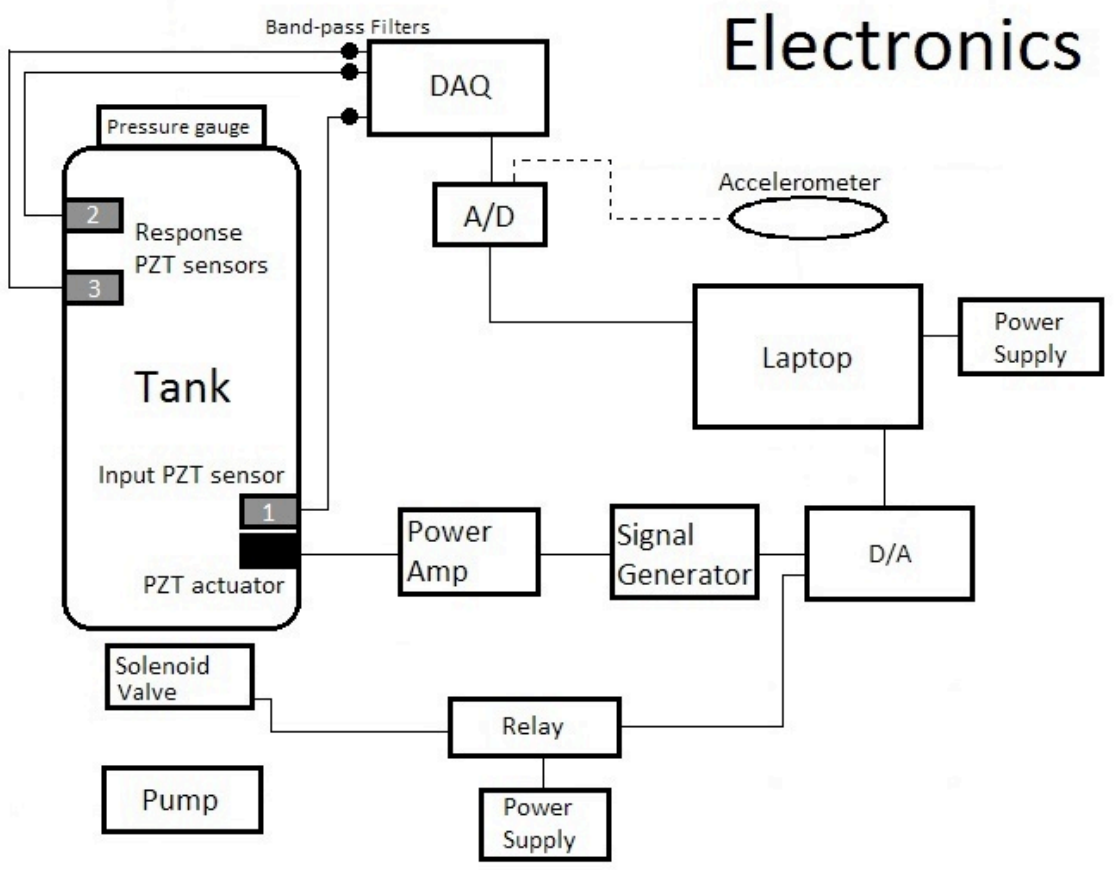

Figure 2: Data acquisition schematic.

terested in the $f_{1 m n}$ modes for comparison with our experimental modes.

For the material and geometric properties of our tank, we find the theoretical modes associated with the equivalent open, simply supported cylinder to be slightly lower than the empty tank modes determined experimentally. The set of theoretical modes for the open cylinder and the closest matching experimental empty-tank modes are shown in Fig. 7 .

While there is reasonable agreement between the lowest experimentally determined modes and the theoretical values for $f_{112}, f_{113}$, and $f_{114}$, these open cylinder modes are generally lower in frequency than the equivalent modes for a tank with end caps. We have verified this by taking FRF data with a model PVC tank with removable end caps. Further, the result is easily understood in terms of the clamping boundary condition provided by the end caps. Such clamping generates fixed nodes that should in- crease the resonant frequencies. Additionally, there are several modes present in the experimental cylinder that do not appear in the theoretical mode spectrum of Soedel. These are likely due to the nodes introduced by mounting hardware, ports, and end-cap resonances.

The key observations associated with Fig. 7 are that (a), the fundamental frequency is not associated with the lowest $n=1$ breathing mode, but is in fact an $n=2$ mode, and (b) the lowest modes observed and tracked in our experiment are associated with the $m=1$ longitudinal mode.

\section{Discussion}

The central objective of the study reported here was to determine the feasibility of using modal analysis to evaluate the fluid load in a spacecraft tank in a reduced gravity environment. Our 1g ground experiments with fluid in static equi- 


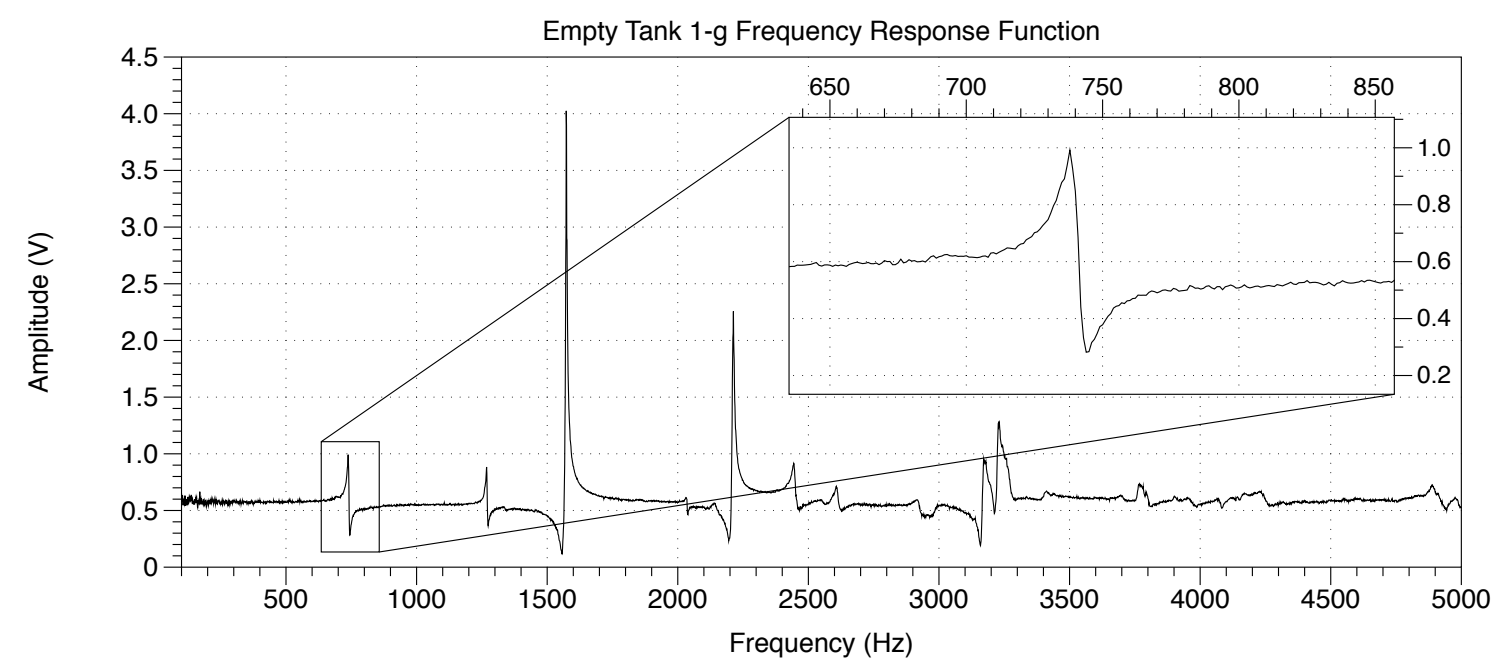

Figure 3: Empty Tank Frequency Response Function. Inset shows blow-up of fundamental mode.

librium revealed the basic mechanism associated with fluid-loading. In these experiments, we determined that the primary effect of fluid loading on the tank was to increase its effective mass rather than its effective stiffness. This is evidenced in the marked reduction of resonant frequency with fill-level. In the 1-g testing, we conclude that modal estimation of fluid volume can achieve a resolution of $5 \%$ or better in the cylindrical portion of the tank. We further determined that the effect of fluid loading on resonant mode shifts was most strongly correlated with the total contact area between fluid and tank wall. We investigated this hypothesis by comparing the mode shifts for a tank in the vertical orientation to those in a tank experiencing equivalent fluid volume changes in the horizontal orientation. Effective contact area changes are smaller for equivalent fluid volume changes in the horizontal orientation, and corresponding mode shifts are reduced as well.

In the reduced gravity environment provided by the parabolic flights, sloshing of the fluid produces continuous variation in effective surface wetting, and so resonant modes are not fixed in this dynamic environment in which the fluid never has time to reach its zero-g free surface configuration. The central question in this case was whether the mode shifts would be large enough to reduce the resolution of the modal analysis technique below the threshold of viability. Our experiments suggest that even in the dynamic sloshing environment of parabolic flight, mode shifts can be averaged over to produce fluid volume estimates with relatively low standard errors.

We have demonstrated that EMA is a viable technique for the real-time, non-invasive estimation of fluid volumes in propellant tanks in microgravity environments. The data presented here suggests that the EMA technique may produce fluid volume resolutions that match or exceed those of existing methods. Given that EMA methods are relatively inexpensive to implement, do not require modification of tank structures, and can provide real-time monitoring of fluid volume in propellant tanks, further studies seem warranted to probe the potential of the methods for addressing the significant and expensive problem of propellant volume gauging in microgravity. 


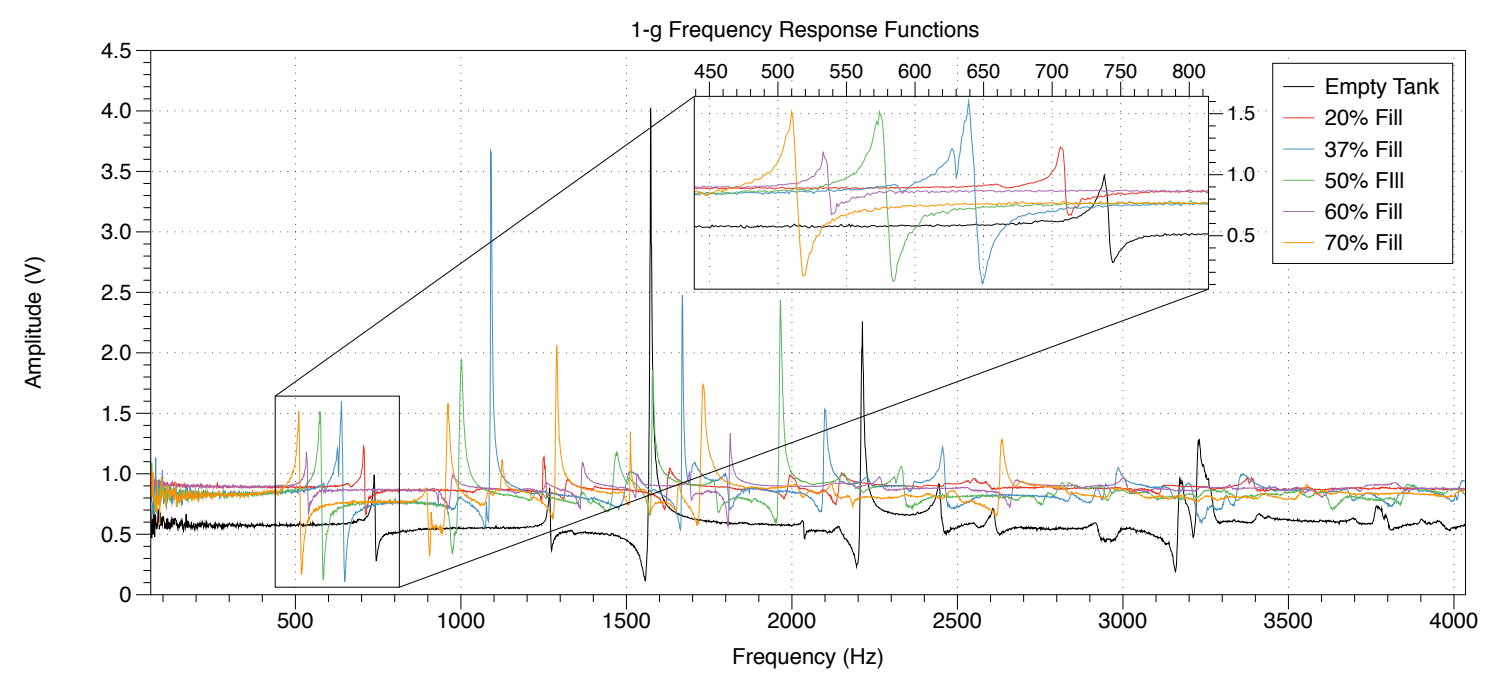

Figure 4: 1-g Frequency Response Functions recorded for fluid in equilibrium with tank in vertical position. Inset shows blow-up of fundamental mode and demonstrates the decrease of mode frequency with increasing fill-fraction.

\section{Acknowledgments}

The authors gratefully acknowledge the Wisconsin Space Grant Consortium for financial support, and the Reduced Gravity Office at NASA Johnson Space Center for support of the Systems Engineering Educational Discovery (SEED) program. The authors also acknowledge the useful contributions made by Prof. Douglas Arion. The Carthage Microgravity Team student members who worked on this project include KelliAnn Anderson, Amber Bakkum, Stephanie Finnvik, Erin Gross, Cecilia Grove, Steven Mathe, Kimberly Schultz, and Danielle Weiland.

\section{References}

[Fortescue et al., 2003] Fortescue, P., Stark, J. \& Swinderd, G. (Eds.). (2003). Spacecraft Systems Engineering, 3rd Ed.. Wiley.

[Malhotra et al., 2000] Malhotra, P., Wenk, T. \& Wieland, M. (2000). Simple Procedure for Seismic Analysis of Liquid-Storage Tanks. Structural Engineering International 3, 197-201.

[National Instruments, 2011] National Instruments, Inc., Austin, TX.

[Pandey et al., 1991] Pandey, A.K., Biswas, M. \& Samman, M.M. (1991). Damage detection from changes in curvature mode shapes. Journal of Sound and Vibration, 145(2), 321-332.

[Peters, 2004] Peters, J. (2004). Spacecraft Systems Design and Operations. Kendall Hunt Publishing.

[Smart Materials, 2011] Smart Materials, Inc., Sarasota, FL.

[Soedel, 1981] Soedel, W. (1981). Vibrations of Shells and Plates. Marcel Dekker Inc.

[Viar, 2011] Viair, Inc., Irvine, CA. 


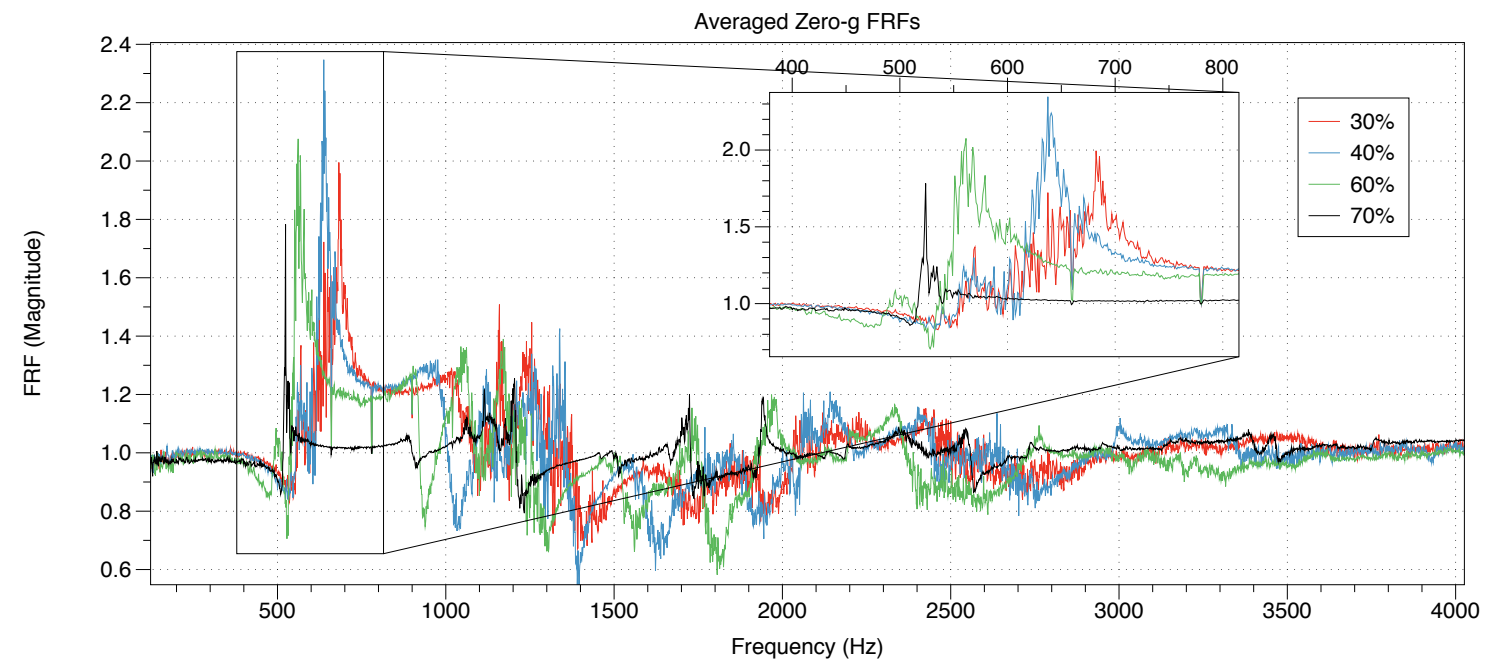

Figure 5: Zero-g Frequency Response Functions recorded for fluid with tank in vertical position. FRFs are averaged over multiple 1.0 second windows. Inset shows blow-up of fundamental mode and demonstrates the decrease of mode frequency with increasing fill-fraction.

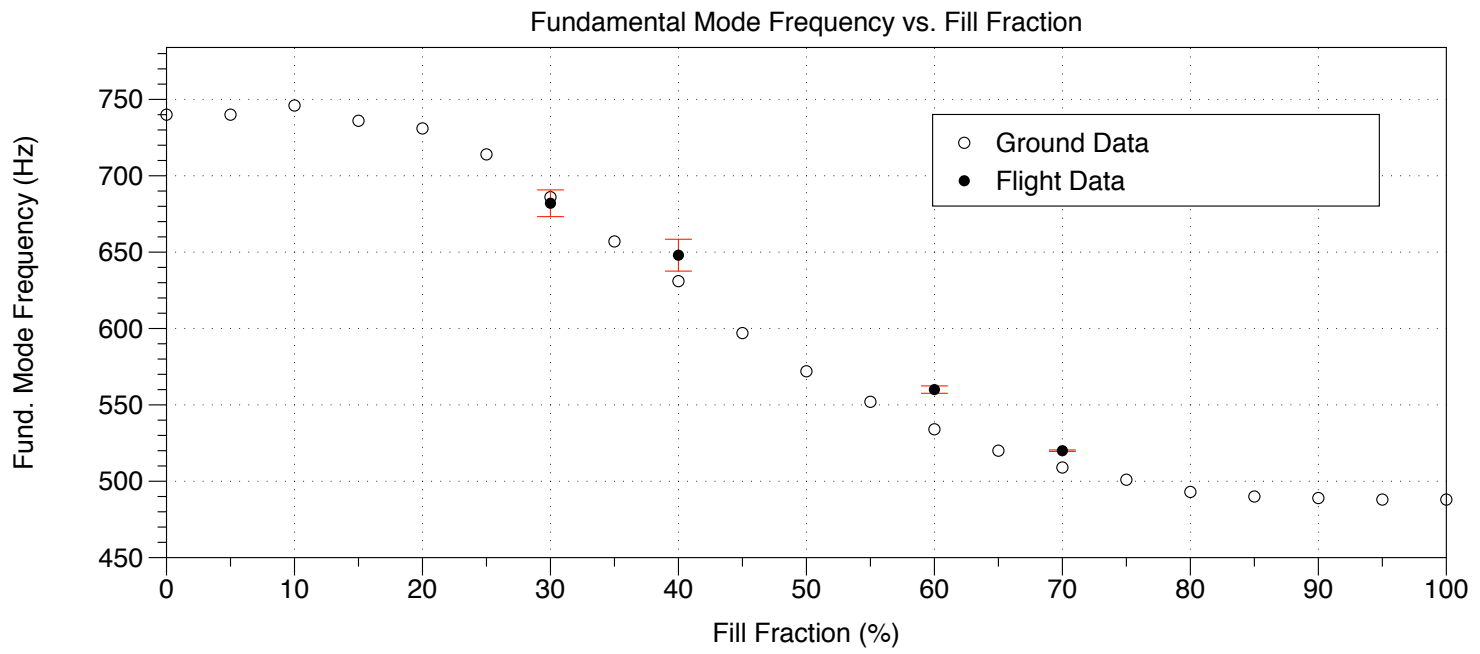

Figure 6: Variation of fundamental mode frequency with fill fraction for both 1-g equilibrium fluid configurations and zero-g slosh-averaged fluid. Error bars on the flight data represent standard error in the data, while error bars for the $1.0 \mathrm{~g}$ data are not depicted, but would be smaller than data symbol. 


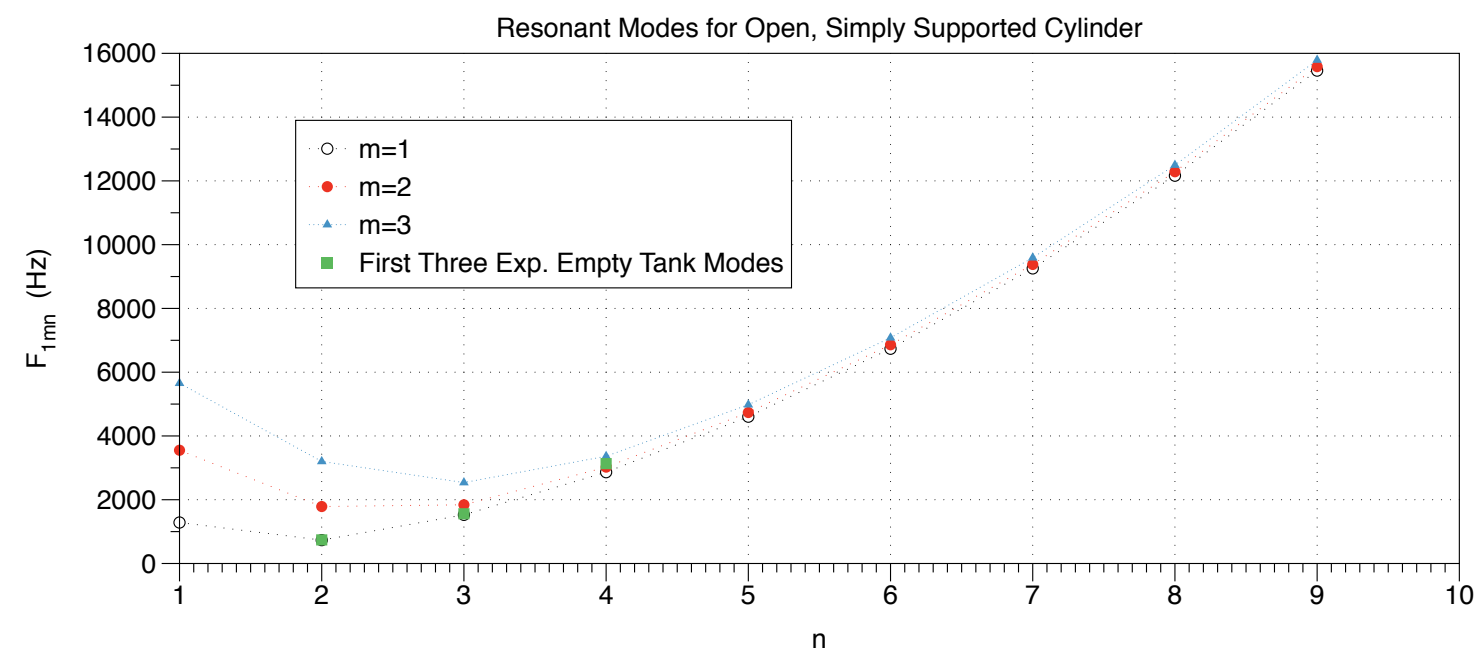

Figure 7: Theoretical modes for the open-ended, simply supported cylinder as derived from Soedel's theory. Also shown are the closest matching experimental modes for the empty tank. 